POS PROCEEDINGS

\title{
Noncommutative Geometry and Physics
}

\author{
Ali Chamseddine* \\ American Univ. of Beirut and Inst. des Hautes Etudes Scientifiques \\ E-mail: chamseaub.edu. lb
}

Classification of finite noncommutative spaces points uniquely to the standard model provided the connection is linear. Relaxing the constraint of linearity allows for the Pati-Salam model of unification of quarks and leptons.

Frontiers of Fundamental Physics 14 - FFP14,

15-18 July 2014

Aix Marseille University (AMU) Saint-Charles Campus, Marseille

${ }^{*}$ Speaker. 